\title{
Bilateral Crystalline Corneal Deposits as First Clinical Manifestation of Monoclonal Gammopathy: A Case Report
}

\author{
Johannes Steinberg ${ }^{\mathrm{a}}$ Mau-Thek Eddy ${ }^{\mathrm{a}}$ Toam Katz $^{\mathrm{a}}$ \\ Eike Matthiessen $^{\mathrm{b}}$ Otto H. Fricke ${ }^{\mathrm{a}}$ Gisbert Richard ${ }^{\mathrm{a}}$ \\ Stephan J. Linke
}

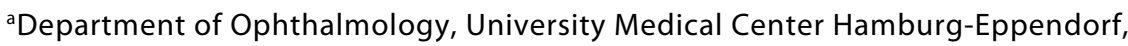
and ${ }^{b}$ Private Practice, Hamburg, Germany

\section{Key Words}

Bilateral corneal deposits · Monoclonal gammopathy · Diagnosis

\begin{abstract}
Aims: To report the clinical and diagnostic findings of a patient with bilateral corneal deposits caused by an underlying monoclonal gammopathy.

Methods: Slit-lamp biomicroscopy, confocal microscopy and additional serological tests were performed on a 35-year-old man presenting with bilateral crystalline corneal deposits.

Results: The patient was diagnosed as having monoclonal gammopathy based on elevated levels of serum immunoglobulin G. Confocal microscopy showed highly reflective (protein) deposits throughout the entire cornea, with the highest density in the epithelium and anterior stromal keratocytes.

Conclusions: Monoclonal gammopathy, a potential sign of a life-threatening disease, can lead to dense, bilateral corneal deposits. As such changes can occur long before ocular or systemic discomforts appear, an early diagnosis is crucial. Ophthalmologists should be aware of corneal deposits as potential warning signs of monoclonal gammopathy.
\end{abstract}

\section{Introduction}

A 35-year-old white man was referred to our outpatient department for further examination after a routine evaluation had demonstrated bilateral corneal opacities. According to the referring ophthalmologist, the corneal findings had remained 
unchanged since the first presentation of the patient 18 months before. No systemic disease had been previously diagnosed.

\section{Materials and Methods}

The evaluation and management of this patient consisted of anamnesis, visual acuity testing, slitlamp microscopic examination of the anterior segment and indirect funduscopic examination. Both corneas were examined with laser scanning in vivo confocal microscopy (Heidelberg Retina Tomograph II, Rostock Cornea Module; Heidelberg Engineering $\mathrm{GmbH}$ ). To differentiate between the possible diagnosis of ocular cystinosis and other systemic causes of protein deposits, additional serological tests, including electrophoresis, were performed, followed by a bone marrow puncture.

\section{Results}

Anamnesis revealed mild glare sensitivity and a slightly reduced contrast sensitivity without any systemic alterations. On examination, best corrected visual acuity was 20/25 in the patient's right eye and 20/20 in his left eye, with an underlying myopia of -7.5 in the right eye and -6.5 in the left eye. Slit-lamp examination revealed one pathologic finding: bilateral, white, crystalline-like opacities throughout the entire depth of the cornea (fig. 1). No difference in the opacities, neither between central and peripheral tissue, nor between the right and left eye, could be seen. Laser scanning in vivo confocal microscopy confirmed a massive amount of highly reflective crystalline deposits throughout all corneal layers (fig. 2 fig. 3 ). The corneal epithelial cells and anterior stromal keratocytes revealed the highest intracellular reflectivity of the deposits.

A hemogram verified a massive increase of immunoglobulin $\mathrm{G}(\operatorname{IgG})(3,775 \mathrm{mg} / \mathrm{dl})$, while serum protein electrophoresis demonstrated a monoclonal IgG kappa band. Based on these results, a bone marrow puncture was performed and the analyses revealed smoldering myeloma. Smoldering myeloma is defined by a monoclonal IgG serum protein of $>3 \mathrm{~g} / \mathrm{dl}$ and/or $>10 \%$ bone marrow plasma cells but with no evidence of endorgan damage (hypercalcemia, renal insufficiency, anemia or bone lesions) [1].

\section{Discussion}

There is a broad variety of single case reports of corneal involvement in systemic hyperparaproteinemia [2]. Meesmann, in 1934, was the first to report corneal deposits in a patient with Bence-Jones proteinuria. Prospective studies concerning the incidence of corneal involvement in monoclonal gammopathies have found only a very low incidence [3]. Corneal involvement in monoclonal gammopathy can present in a variety of ways. Usually, changes occur bilaterally due to a systemic cause. The precise etiology of corneal immunoprotein deposition in the setting of paraproteinemias has been discussed in the literature. Regarding the involved layers, all facets are possible: from deposits only affecting the epithelial layer to deeper, stromal involvement. It is assumed that the different locations of the abnormal immunoglobulin deposits reflect the differences in the origin of the paraprotein. Paraprotein transport via the tear film, diffusion from aqueous fluid from the anterior chamber and influx via the perilimbal vascular arcades are the three mechanisms, either working alone or in combination, which comprise the most 
postulated theories $[4,5]$. Immunoglobulin deposits in the cornea can be exclusively intracellular, extracellular or a combination of both types [6].

Due to the rarity of this phenomenon, patients without a known systemic disease who present with diffuse corneal alterations are often misdiagnosed. Examples of differential diagnoses include lattice dystrophy, Schnyder's crystalline dystrophy, deep filiform dystrophy and ocular cystinosis [2]. The very precise symmetrical pattern of the deposits with regard to distribution within different corneal layers (revealed by confocal microscopy; compare fig. 2 and 3) might be helpful in diagnosing a systemic disease as causative for the corneal alterations. Regarding treatment options and the prognosis of the corneal involvement, there is very little information in the literature. Many of the presented cases are associated with only minimal visual symptoms and require no specific ocular therapy. Control of the systemic condition usually improves the ocular symptoms in these patients [2]. In the very few cases of severe and lasting corneal involvement, penetrating keratoplasty seems to be the treatment of choice [7].

\section{Disclosure Statement}

The authors have no proprietary interests and no financial support was received for this paper. No conflicts of interest regarding this paper exist.

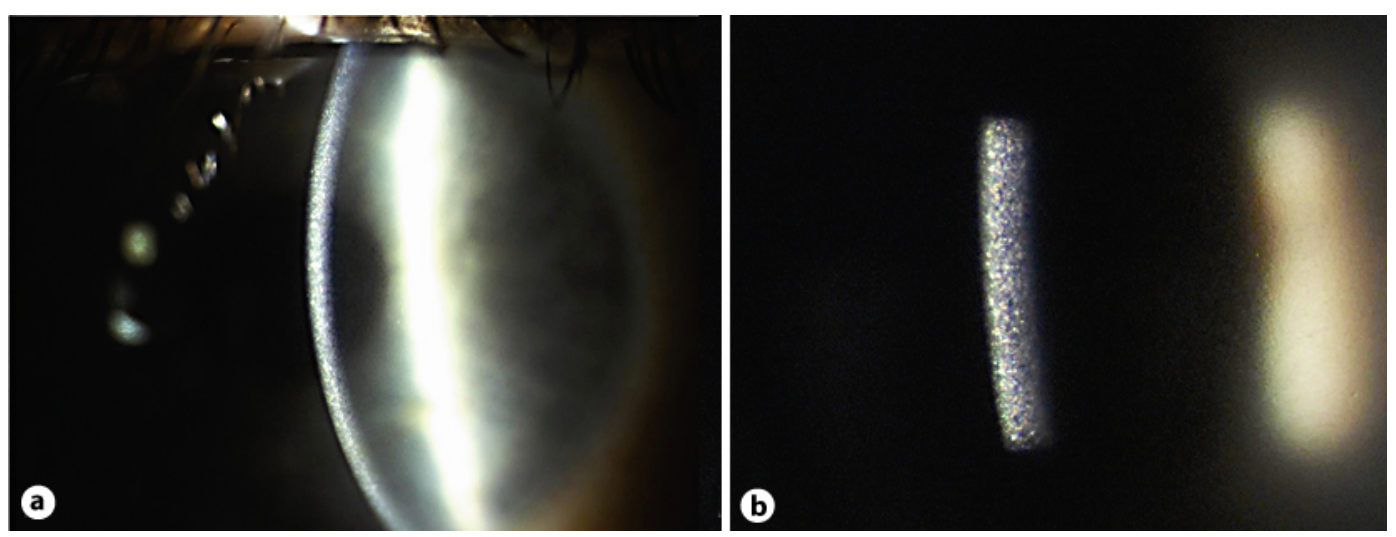

Fig. 1. Slit-lamp biomicroscopy reveals greyish-white deposits in the entire cornea of both eyes. a Magnification $\times 16$. b Magnification $\times 25$. 

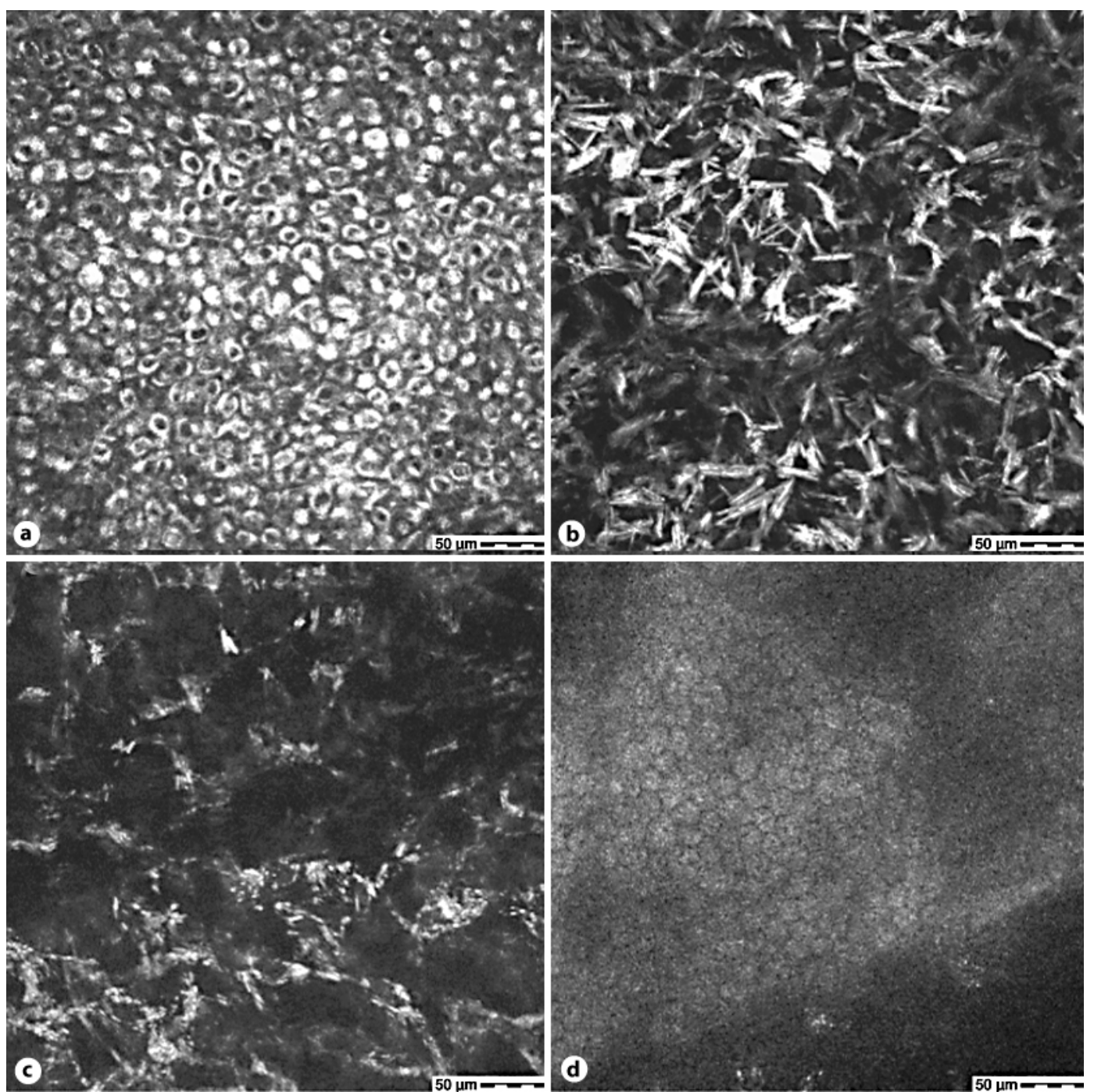

Fig. 2. Confocal microscopy of the cornea (right eye) shows highly reflective intracellular deposits in the epithelium (a): depth $5 \mu \mathrm{m}$, anterior stromal keratocytes (b): depth $52 \mu \mathrm{m}$ and mid stromal keratocytes (c): depth $354 \mu \mathrm{m}$. d The endothelium appears regular in morphology and without significant intracellular deposits. 

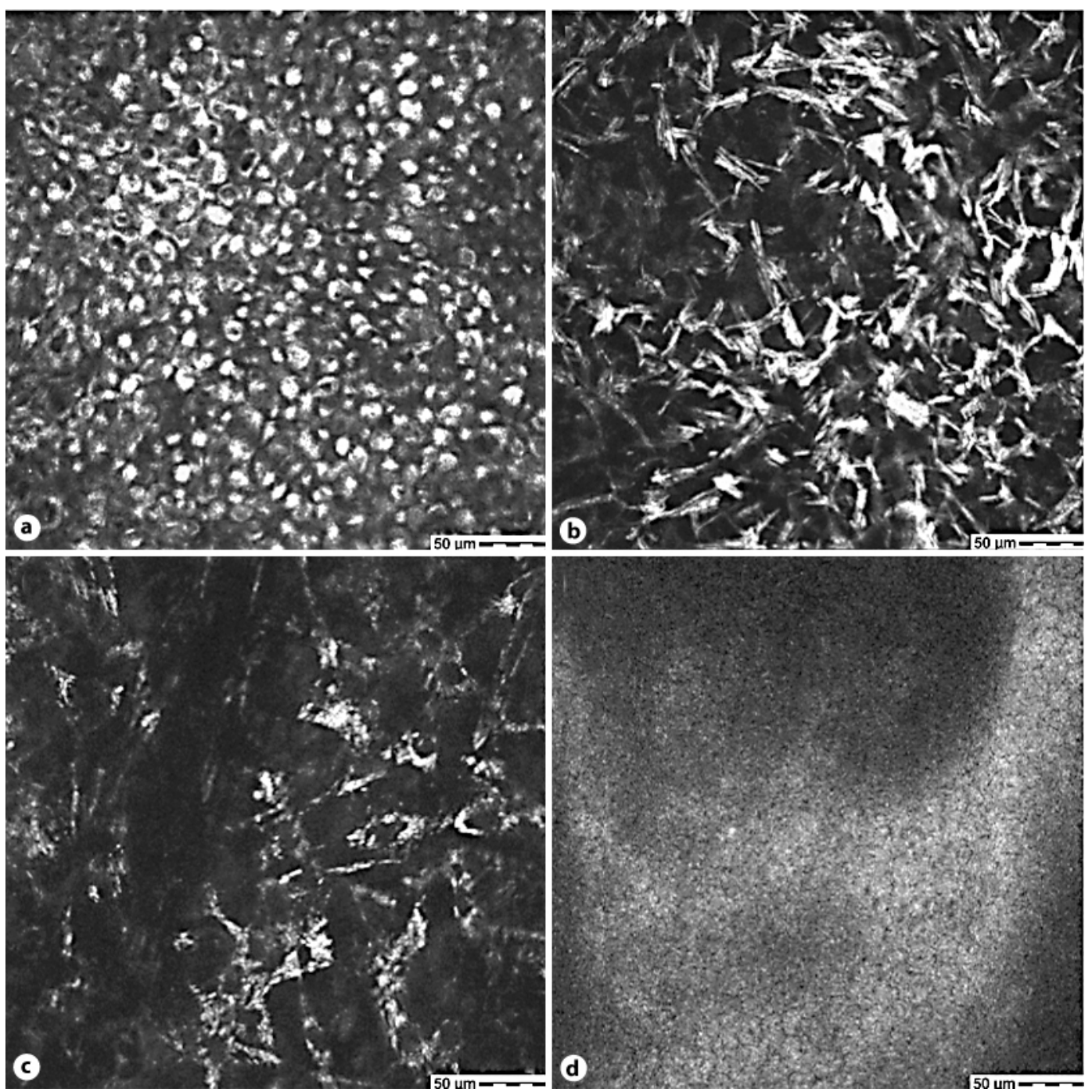

Fig. 3. Confocal microscopy of the cornea (left eye) shows highly reflective intracellular deposits in the epithelium (a): depth $4 \mu \mathrm{m}$, anterior stromal keratocytes (b): depth $49 \mu \mathrm{m}$ and mid stromal keratocytes (c): depth $348 \mu \mathrm{m}$. d The endothelium appears regular in morphology and without significant intracellular deposits. 


\section{References}

1 IMWG: Criteria for the classification of monoclonal gammopathies, multiple myeloma and related disorders: a report of the International Myeloma Working Group. Br J Haematol 2003;121:749-757.

2 Garibaldi DC, Gottsch J, de la Cruz Z, Haas M, Green WR: Immunotactoid keratopathy: a clinicopathologic case report and a review of reports of corneal involvement in systemic paraproteinemias. Surv Ophthalmol 2005;50:61-80.

3 Bourne WM, Kyle RA, Brubaker RF, Greipp PR: Incidence of corneal crystals in the monoclonal gammopathies. Am J Ophthalmol 1989;107:192-193.

-4 Kato T, Nakayasu K, Omata Y, Watanabe Y, Kanai A: Corneal deposits as an alerting sign of monoclonal gammopathy: a case report. Cornea 1999;18:734-738.

-5 Schelonka LP, Ogawa GS, O'Brien TP, Green WR: Acute unilateral corneal immunoprotein deposition in IgM monoclonal gammopathy. Arch Ophthalmol 2000;118:125-126.

6 Barr CC, Gelender H, Font RL: Corneal crystalline deposits associated with dysproteinemia. Report of two cases and review of the literature. Arch Ophthalmol 1980;98:884-889.

7 Font RL, Matoba AY, Prabhakaran VC: IgG-kappa immunoglobulin deposits involving the predescemetic region in a patient with multiple myeloma. Cornea 2006;25:1237-1239. 